\title{
Sarcoma of the Lower Limb: Reconstructive Surgeon's Perspective
}

\author{
Zhixue Lim ${ }^{1}$ Sophia A. Strike ${ }^{1}$ Mark E. Puhaindran ${ }^{1}$ \\ ${ }^{1}$ Department of Hand and Reconstructive Microsurgery, National \\ University Hospital, Singapore, Singapore
}

\begin{abstract}
Address for correspondence Zhixue Lim, MRCS (Edin), Department of Hand and Reconstructive Microsurgery, National University Hospital, NUHS Tower Block, Level 11, 1E Kent Ridge Road, Singapore 119228 , Singapore (e-mail: zhixue.lim@mohh.com.sg).
\end{abstract}

\begin{abstract}
Keywords

- sarcoma

- lower extremity

- reconstruction

- vascularised bone graft

- soft tissue reconstruction

Management of sarcomas in the lower extremities have evolved from amputations to limb-preserving surgeries with evidence to support that they have equal overall survival, albeit with better functional outcome. The challenge of reconstruction lies in providing a durable, functional, and aesthetically pleasing limb. However, limbpreserving intention should not delay interventions that provide a survival benefit such as chemotherapy and radiotherapy. The advent of radiotherapy and chemotherapy also has implications on wound healing and should be considered during the reconstructive process. This article reviews the methodical approach, reconstructive strategies, and considerations for the reconstructive surgeon with respect to the lower extremity after sarcoma excision.
\end{abstract}

\section{Introduction}

Sarcomas are a diverse group of neoplasms that account for approximately $1 \%$ of adult malignancies and 7 to $15 \%$ of pediatric malignancies. ${ }^{1}$ The range of histological subtypes contributes to the varied prognoses. The majority of extremity sarcomas occur in the lower extremity ( 74 vs. $26 \%$ in the upper limb), ${ }^{2}$ translating to higher rates of lower limb reconstruction after sarcoma excision. The considerations for reconstruction of the lower limb differ greatly from that of the upper limb due to weight bearing requirements, higher risk of infectious complications, and slower wound healing in the lower limb. This article will review the history of sarcoma treatment and current methods of skeletal and soft tissue reconstruction.

\section{Evolution of Sarcoma Treatment}

In the past, the cornerstone of sarcoma treatment was amputation, achieving relatively low recurrence rates at the expense of functional integrity. In 1879, Samuel Gross shared his experience with 165 sarcoma cases, in which he advocated early amputation, despite reporting an operative mortality of up to $30 \%{ }^{3}$ Gross recommended early amputation since he noted inevitable recurrence in the cases that were treated with limb-salvage. However, some surgeons persisted and continued to refine their techniques, including Phemister who described a systematic approach to limb-preserving surgery in his article "Conservative surgery in the treatment of bone tumours," where he shared his personal experience in combining radiotherapy and surgical excision, with the possibility of bone transplantation after resection of bone sarcomas. He highlighted that limb-sparing surgery may achieve similar recurrence free rates as amputations. ${ }^{4}$

In more recent times, the advent of chemotherapy and radiotherapy, coupled with a marked improvement in diagnostic imaging technology, has revolutionized the management of most tumors, including sarcomas. The improved clinical outcomes from this multimodality approach precipitated the rise of limb-salvage surgery, which brought about novel reconstructive methods in a bid to maintain a durable, functional, and aesthetically pleasing option for patients. Although limb-salvage has a higher local recurrence rate than amputation, overall survival is equal. ${ }^{5,6}$

The benefit of radiotherapy is well established in the management of large soft tissue sarcomas. Chemotherapy is not routinely used for soft tissue sarcomas but can play a role for large sarcomas of the trunk and extremities, and for a small select group of soft tissue sarcomas that are known to respond to chemotherapy. On the other hand, chemotherapy is routinely used for osteosarcomas and Ewing's sarcomas. In a cancer database of 3,422 patients with large extremity or truncal sarcoma, radiotherapy was shown to decrease
Surgeons of India
License terms

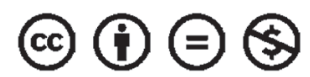


the hazard ratio of death by $34 \%$ and chemotherapy by $24 \%$ when compared with surgery alone. ${ }^{7}$ Compared with surgery alone, multimodal treatment improves disease-free survival in osteosarcoma patients from 10 to $20 \%$ to $>60 \% .{ }^{8}$ O'Bryan et al were also the first to show a dose-dependent response with doxorubicin when used on patients with soft tissue sarcoma. ${ }^{9,10}$

Coordinated multimodal local therapy is critical in ensuring recurrence-free limb sparing surgery. Radiotherapy has been shown to decrease local recurrence rates in limb-salvage procedures. ${ }^{6}$ This adds a different facet to the consideration of wound healing complications during reconstructive surgery, as there are more wound-related complications when radiotherapy is used. ${ }^{11,12}$ The choice and timing of radiotherapy also have a significant impact on wound healing post-coverage, with intensity modulated radiotherapy achieving better outcomes compared with three-dimensional conformal radiotherapy. ${ }^{8}$ There is also a evidence to suggest that neoadjuvant radiotherapy imposes a greater risk of wound complications compared with adjuvant radiotherapy. ${ }^{13}$

The field of sarcoma treatment is evolving, with progressively more aggressive attempts at lower extremity limb-salvage with the availability of more efficacious adjuvant treatment. This is a summary of the reconstructive options that can be utilized to facilitate limb-salvage.

\section{Reconstruction Options}

\section{Skeletal Reconstruction}

The basis of ensuring a stable reconstruction in the lower limb begins with skeletal reconstruction. A bone defect, when present, often poses a huge challenge to the reconstructive surgeon. To tackle the issue, one has to take various factors into account such as (1) the size of the bony defect, (2) the vascularity of the soft tissue bed, (3) the adequacy of soft tissue cover, and (4) the eventual function of the limb. ${ }^{14}$

The various options for the reconstruction of diaphyseal bone loss are nonvascularized cancellous and cortical autografts, vascularized bone autograft, cadaveric bone allografts or a combination thereof, induced-membrane, and bone transport techniques (isolated shortening, compression followed by distraction and segmental bone transport), ${ }^{15}$ biological reconstruction with a combination of techniques including autoclaved autograft bone and endoprosthetic reconstruction.

For substantial bone defects $(>10 \mathrm{~cm})$, segmental bone transport as initially described by Ilizarov, ${ }^{16}$ and later refined by Cattaneo et al, ${ }^{17}$ can also be considered,

\section{Nonvascularized Bone Graft}

In 1983, Weiland et al reviewed their series of 41 vascularized bone grafts (VBG) and found that for long bone defects (> 6cm), VBG was conferred a significant advantage. ${ }^{18}$ This $6 \mathrm{~cm}$ rule has since proliferated and later reviews on long bone reconstructions have also adhered to the above rule. In general, there is no compelling evidence that VBGs produce better outcomes for longer bone defects. There are studies that demonstrate that VBGs result in better radiographic and functional outcomes ${ }^{19-21}$; however, these studies did not examine grafts based on length. Given that there is no strong evidence mandating one over the other, the choice of bone grafting remains surgeon dependent. Considerations such as operative time, patient factors, and burden of microvascular surgery should be kept in mind.

\section{Vascularized Bone Graft}

VBGs can be useful in scenarios where there is a large bony defect, especially when compounded with issues of infection, scarring, and poor vascularity.22 They are also superior in strength, remodeling, and hypertrophy. In a series out of Memorial Sloan-Kettering Cancer Centre with 10-year follow-up data, Chen et al showed that the microvascular free fibular flap has a lower infectious complication rate and higher rate of bone union when compared with the traditional allograft. ${ }^{23}$

Typically, microvascular free fibular VBG is the most suitable in lower limb reconstruction due to its shape, vascular pedicle predictability, and potential for hypertrophy. A commonly encountered conundrum is that the size of the defect may not be compatible with that of the vascularized fibular graft. The tibia, on one hand, may prove a good match for a vascularized fibula, while the femur, which has a much larger cross-sectional area, may be predisposed to stress fracture when reconstructed with a vascularized fibula. However, the versatility of being able to fold a single vascularized fibular graft into two segments, to use two free fibulas, or the ability to combine a vascularized fibular graft with an allograft can help to address this issue. ${ }^{24}$ - Fig. 1 demonstrates an example of a young patient with Ewing's sarcoma who underwent resection of proximal femur and subsequent reconstruction with vascularised fibular graft and iliac crest bone graft, fixed with an angled blade plate. Follow-up radiographs demonstrate good union and stability of reconstruction.

\section{Composite Vascularized Bone Graft with Allograft}

A size disparity reduces the contact area with the recipient bone and potentially causes higher rates of mechanical failure and fracture. The addition of allograft allows for short-term mechanical stability, while allowing for biological activity from the VBG to take effect in the long term. This modality is more suitable for tumor reconstruction due to the propensity of allografts to infection, and hence may be relatively contraindicated in cases of osteomyelitis or open fractures. ${ }^{25}$

Capanna et al described composite free vascularized fibular grafts with massive allografts in their 1993 series of reconstructions of large metadiaphyseal bone defects. Results were promising in this study of 14 cases, with no displaced fractures and all patients walking without external aids on follow-up at 2 to 5 years. ${ }^{26}$

\section{Biological Reconstruction: Autoclaved Autograft Bone Combined with Vascularized Bone and Bone Marrow} In a study of 100 rabbits, Taguchi et al demonstrated that autoclaved autograft bone could enhance bone union when supplemented with autogenous bone marrow and a VBG. This translated to better torsional stiffness. ${ }^{27}$ Puhaindran 

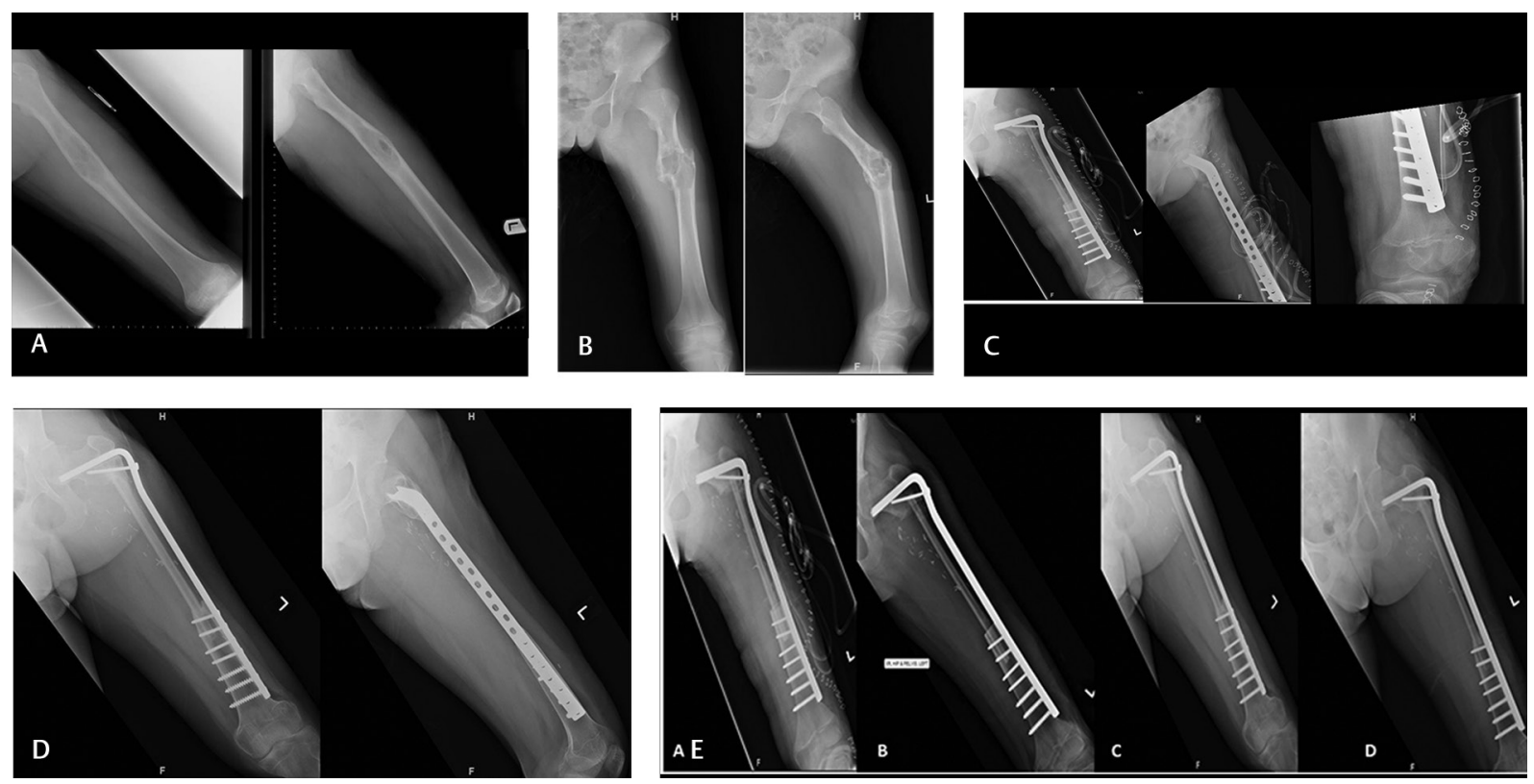

Fig. 1 (A) A 13-year-old patient who presented with a lytic lesion in the left femur and subsequently underwent curettage of lesion. (B) Curettage histology confirmed Ewing's sarcoma (performed at a different center)-radiographs (post-curettage) when patient first presented to us. (C) Postoperative day 1 radiograph-complete resection of left proximal femur, reconstruction of bone defect with vascularized fibular graft, and iliac crest bone graft. Subsequent fixation with an angled blade plate. (D) Postoperative radiograph at 2 years. (E) Comparison of radiographic progression of union: (A) Immediate post-reconstruction, (B) 2 months post-reconstruction, (C) 1.5 years post-reconstruction, (D) 2 years post-reconstruction.

and Pho developed a biological approach based on the above principles, where large bone defects in children were bridged by vascularised fibular graft supplemented with autoclaved autologous bone grafts infused with bone marrow, to achieve immediate stability, early weight bearing and rehabilitation. ${ }^{28}$

In a series of nine children treated with the above method (seven tumors of the distal femur, two tumors of the proximal tibia), none of the five surviving patients required revision surgery from failure of mechanical construct. ${ }^{28}$ In our center, we have had the benefit of following up these patients for up to three decades. The durability, functional, and cosmetic outcomes have been remarkable. - Fig. 2 displays an example of a patient with osteosarcoma of the distal femur, who underwent resection and subsequent reconstruction with VBG and autoclaved bone graft.

While some surgeons may still opt for allograft, the benefits of lower cost, anatomical matching, and absence of graft versus host immune reactions have made autoclaved bone graft an attractive option.

\section{Endoprosthesis}

Endoprosthetic replacement for bone sarcomas of the lower extremity is also well established with several studies exhibiting satisfying functional results in patients with longterm survival. Schwartz et al reviewed 489 patients after resection of musculoskeletal tumors and reconstruction using an endoprosthesis. No cases of amputation were performed as a direct outcome of mechanical failure. There was also evidence that new modular systems outperformed the custom designs ( $90.8 \%$ survival and $59.6 \%$ survival). ${ }^{29}$

Given that the survival of segmental endoprostheses is a concern (10-year survival rates of segmental endoprostheses

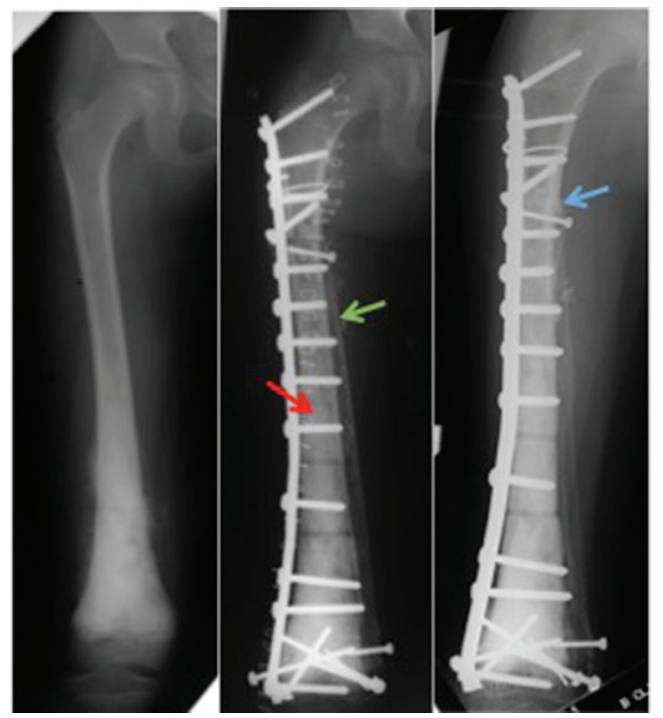

Fig. 2 Vascularized fibular graft (green arrow) with autoclaved bone graft (red arrow) and plate fixation in a patient with osteosarcoma of the distal femur. Evidence of union between native femur, autoclaved bone graft, and vascularized bone graft (blue arrow) on 1 year follow-up.

ranges from 63 to $80 \%$ ), ${ }^{30,31}$ this option is typically used in elderly patients or patients with poor prognoses, where immediate weight bearing and function outweigh durability of the construct. It can also be extremely useful in alleviating pain or correcting instability in patients with metastatic bone disease, myeloma, or lymphoma. ${ }^{32}$ While endoprosthetic replacements provide patients good functional recovery, one has to weigh this against the feared complications of fracture (prosthetic or periprosthetic), infection, and aseptic loosening. 30,33,34 


\section{Rotationplasty}

While the outcomes for major reconstructive procedures have improved in advanced disease, limb-salvage may not always be possible. The rotationplasty serves as an alternative option for sarcomas of the lower extremity. As early as 1922, Sauerbruch ${ }^{35}$ described "Umkippplastik," today's equivalent of rotationplasty, Salzer et al first reported the use of rotationplasty for sarcomas of the lower extremity. ${ }^{36}$ Hopyan et al have found superior Musculoskeletal Tumour Society (MSTS) scores compared with above-knee amputees, but poorer scores compared with limb-salvage. ${ }^{37}$ However, the long-term outcomes have proven rotationplasty to be an alternative that allows patients to stay active, with mean walking speeds of $54.2 \mathrm{~m} / \mathrm{min}$ compared with $77.4 \mathrm{~m} / \mathrm{min}$ in healthy controls. ${ }^{38}$ The main impediment of the procedure has been argued to be psychosocial. In a study of 70 patients with malignant tumors treated with rotationplasty, Gottsauner-Wolf et al found that patients were satisfied with the procedure that they had chosen, with only one patient diagnosed with depression and reported sexual dysfunction post-surgery. ${ }^{39}$ The lower complication rates and good functional outcomes contribute to patient satisfaction in this modality of treatment. Therefore, where limb-salvage is contraindicated, rotationplasty remains an attractive option.

\section{Vascular Reconstruction}

Sarcomas can encroach on major vessels, making their sacrifice necessary during tumor resection. Following this, arterial reconstruction is mandatory to keep the limb viable. This can usually be performed using an autologous saphenous vein graft or a synthetic graft.

On the other hand, the evidence of venous reconstruction may not be so clear despite it being able to reduce risks of long-term post-thrombotic syndrome. ${ }^{40,41}$ Nishinari et al retrospectively reviewed 44 revascularization procedures in soft tissue sarcomas in the lower extremities; he found that vascular occlusion rates were not significantly higher between arterial and venous reconstructions. The occlusion rate was only significantly higher when synthetic grafts were used compared with autologous saphenous vein grafts. ${ }^{42}$ In terms of development of venous insufficiency, Tsukushi et al found that there were no discernable differences with respect to significant edema, complications, or postoperative function when comparing arterial reconstruction to arteriovenous reconstruction. ${ }^{43}$
Given the lack of compelling evidence for venous reconstruction, the decision to proceed with venous reconstruction lies with the surgeon. Nevertheless, the manifestations of limb swelling, claudication, pigmentation, and eczema can typically be managed conservatively to good effect, such as limb elevation and compression stockings. ${ }^{41,44}$

\section{Soft Tissue Reconstruction}

Some of the options available in a reconstructive surgeon's armamentarium include skin grafts, local-regional flaps, and free flaps for soft tissue coverage; tendon transfers and muscle transfers (pedicled or free) for the restoration of motor function.

\section{Skin Grafts}

If a wound cannot be closed primarily or cannot be allowed to heal by secondary intention, a skin graft is a fast, reliable, and safe option. This is guided by the size and the type of tissues exposed, Skin grafts can also be used in conjunction with the other forms of reconstruction.

\section{Locoregional Flaps}

A variety of local flaps can be selected for soft-tissue coverage. A local pedicled flap is a good choice for reconstruction in small to mid-sized defects that cannot be closed primarily or skin grafted. Parrett et al and Pinsolle et al have published evidence from their studies that favor local flaps. ${ }^{45,46}$

\section{Free Flaps}

The advantage of a free flap is that the surgeon can perform a resection with optimal margins, allowing for better local control. Examples of commonly used free flap options include latissimus dorsi, serratus anterior, gracilis, anterolateral thigh, anteromedial thigh, lateral arm, and radial forearm flap. Proponents of free flaps argue that pedicled flaps do not provide sufficient coverage and may introduce a further plane to the dissemination of disease. In a single center study by Cordeiro et al, where 59 free flaps were performed following oncologic resection, of which 35 patients underwent adjuvant therapy; it showed similar complications to free flaps used for other indications. ${ }^{47}$ This shows that free flaps are a reliable option for oncological reconstruction. - Fig. 3 demonstrates an example of a patient with sarcoma of the foot undergoing resection and immediate coverage with a free lateral arm flap.

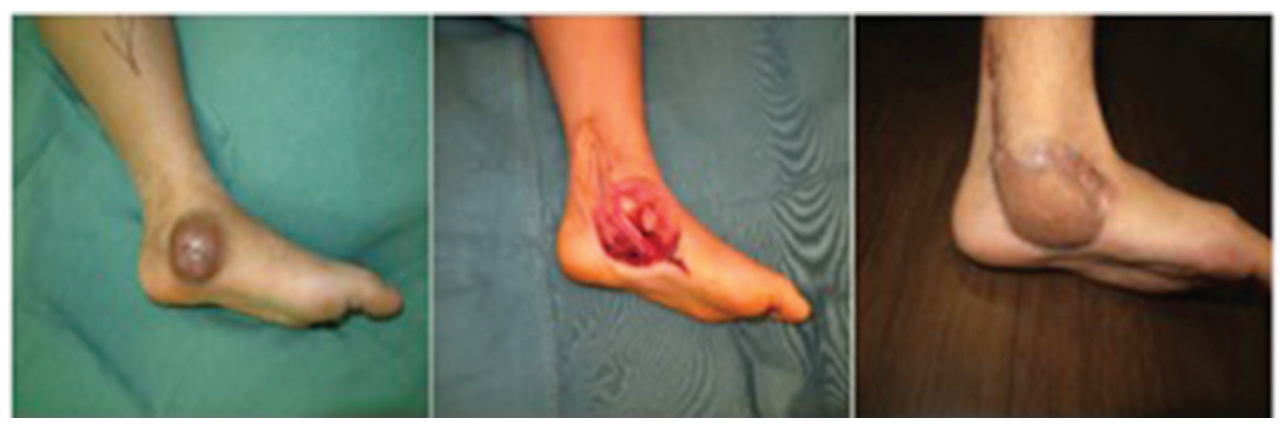

Fig. 3 Patient with clear cell sarcoma of the foot, who underwent resection and immediate free lateral arm flap coverage of the defect. 


\section{Motor Reconstruction}

Radical tumor excision can lead to massive tissue loss, occasionally precluding even local tendon transfers to restore muscle function. The choice for motor reconstruction will depend on biomechanical demands of the recipient site and the familiarity of the surgeon. Commonly used donor muscles include the latissimus dorsi, gracilis, rectus femoris, and tensor fasciae lata. ${ }^{47-53}$

Of particular relevance is the anterior compartment of the thigh, which is the most common site for soft tissue sarcomas. The degree of resection of the quadriceps has been correlated to the decrease in functional ability. Markhede and Stener concluded that isometric knee extension strength was reduced by $22 \%$ after resection of a single component of the quadriceps, $33 \%$ for two components resected, $55 \%$ for three components, and $76 \%$ if more than three were resected. ${ }^{54}$ There are multiple muscle transfers available for restoration of knee extension.

\section{Sartorius Transfer}

Goldthwait in 1987 described the sartorius transfer after quadriceps resection. He discussed that in addition to functional augmentation, the sartorius acts as a vascularized cover allowing connective tissue ingrowth. ${ }^{55}$ However, of the eight patients he studied, all had at least two functional components of quadriceps remaining and hence it is difficult to attribute functional outcomes to the transfer.

\section{Hamstring Transfer}

This method involves detaching the long head of biceps femoris from the distal fibular insertion, anchoring it distally to the patella and tethering it longitudinally to the remains of the quadriceps. Pritsch et al performed a case study in five sarcoma patients and demonstrated that mean power grade of 4.2 and MSTS good or excellent function were achieved after long head of biceps femoris transfer. ${ }^{56}$

\section{Gastrocnemius Transfer}

Initially described by Dubousset et al in $1985,{ }^{57}$ the medial gastrocnemius can be transposed anteriorly in a bipedicled fashion without division of its proximal or distal attachments, with an associated longitudinal tenorrhaphy to the patellar tendon. However, it is difficult to comment on the functional outcome of gastrocnemius transfers as they are often performed with concomitant reconstruction of the proximal tibia, with preservation of part of the quadriceps muscle.

This is akin to other forms of other muscle transfers that have been performed but functional outcomes have not been independently reported. This includes the tensor fascia lata and gracilis.

\section{Free Functioning Latissimus Dorsi Transfer}

Muramatsu et al reported that in cases where three or more components of the quadriceps have been resected, transfer of the latissimus dorsi muscle is indicated as it allows active knee extension to improve to an muscle manual testing (MMT) grading score of 2 after reinnervation of the transferred muscle. There was electromyographic evidence of muscle reinnervation at a mean time of 4.7 months. ${ }^{58}$

\section{Reconstructive Surgeon's Perspective}

Multidisciplinary teams involving the oncology surgeons, medical oncologists, radiation oncologists, radiologists, and pathologists are now the norm when managing sarcoma patients. The reconstructive surgeon should be involved as a part of this team from the beginning. It entails an active process of "pre-neoadjuvant" discussion and meticulous planning where the surgeon discusses potential considerations regarding the reconstructive process-both the type of reconstruction required and the potential donor sites and vascular anastomosis sites. This is especially important in the era of neoadjuvant radiotherapy and chemotherapy. For radiotherapy, it is helpful to discuss and plan the radiation fields with the radiation oncologist, in an effort to limit the field of compromised tissues as well as to plan sites of microvascular anastomosis out of the radiation target zone. For chemotherapy, it would be helpful to understand the timing of chemotherapy and window for surgery, as well as a general understanding of the agents used and their effects on the patient (and surgery). In patients with recurrent tumors, radiotherapy and chemotherapy may have been administered during the prior treatment, and the surgical anatomy may be altered by previous surgery. Reconstruction for a recurrent tumor thus requires an even higher level of meticulous planning given these preoperative obstacles.

Reconstruction is frequently performed at the same time as the surgical resection. At times, it may need to be staged if the patient cannot tolerate a long reconstruction, or if the surgical margins are not certain. This should be anticipated and planned for, and makes it all the more important that the reconstructive surgeon is part of the multidisciplinary team caring for the patient.

It cannot be forgotten that the primary aim of oncological surgery is complete tumor excision with negative surgical margins. Reconstructive priorities cannot supersede this.

\section{Conclusion}

There is no clear algorithm to guide us in reconstruction following lower limb sarcoma resection. We need to tailor treatment to the patient in terms of size of defect, location of defect, requirement for adjuvant therapy, and surgeons' familiarity with the proposed procedure. However, it is even more important for the reconstructive surgeon to be involved in the planning of treatment as part of the multidisciplinary team from the beginning to achieve the best possible outcomes.

\section{Conflict of Interest}

None declared

\section{References}

1 Sondak VK. Sarcomas of bone and soft tissue. In Greenfield LJ, Mulholland MW, Oldham KT, Zelenock GB, Lillemoe KD, eds. Surgery: Scientific Principles and Practice, 3rd ed. Philadelphia: Lippincott Williams \& Wilkins; 2000:2258-2280

2 Weitz J, Antonescu CR, Brennan MF. Localized extremity soft tissue sarcoma: improved knowledge with unchanged survival over time. J Clin Oncol 2003;21(14):2719-2725 
3 Gross SW. The classic: sarcoma of the long bones: based upon a study of one hundred and sixty-five cases. Clin Orthop Relat Res 1975;(111):5-13

4 Phermister DB. The classic: conservative bone surgery in the treatment of bone tumours 1940. Clin Orthop Relat Res 1986; (204):4-8

5 Rougraff BT, Simon MA, Kneisl JS, Greenberg DB, Mankin HJ. Limb salvage compared with amputation for osteosarcoma of the distal end of the femur. A long-term oncological, functional, and quality-of-life study. J Bone Joint Surg Am 1994;76(5):649-656

6 Beane JD, Yang JC, White D, Steinberg SM, Rosenberg SA, Rudloff U. Efficacy of adjuvant radiation therapy in the treatment of soft tissue sarcoma of the extremity: 20-year follow-up of a randomized prospective trial. Ann Surg Oncol 2014;21(8):2484-2489

7 Mahmoud O, Tunceroglu A, Chokshi R, et al. Overall survival advantage of chemotherapy and radiotherapy in the perioperative management of large extremity and trunk soft tissue sarcoma; a large database analysis. Radiother Oncol 2017;124(2):277-284

8 Bielack S, Carrle D, Casali PG; ESMO Guidelines Working Group. Osteosarcoma: ESMO clinical recommendations for diagnosis, treatment and follow-up. Ann Oncol 2009;20(4, Suppl 4):137-139

9 Jacobs EM. Combination chemotherapy of metastatic testicular germinal cell tumors and soft part sarcomas. Cancer 1970;25(2):324-332

10 O'Bryan RM, Baker LH, Gottlieb JE, et al. Dose response evaluation of adriamycin in human neoplasia. Cancer 1977;39(5):1940-1948

11 Prendergast B, Fiveash JB, Gibbs CP, Scarborough MT, Indelicato DJ. Radiotherapy for soft tissue sarcoma of the proximal lower extremity. Sarcoma 2010;2010:829498

12 Sharma K, Marcus JR. Bevacizumab and wound-healing complications: mechanisms of action, clinical evidence, and management recommendations for the plastic surgeon. Ann Plast Surg 2013;71(4):434-440

13 O'Sullivan B, Davis AM, Turcotte R, et al. Preoperative versus postoperative radiotherapy in soft-tissue sarcoma of the limbs: a randomised trial. Lancet 2002;359(9325):2235-2241

14 Gan AW, Puhaindran ME, Pho RW. The reconstruction of large bone defects in the upper limb. Injury 2013;44(3):313-317

15 Uzel A-P, Lemonne F, Casoli V. Tibial segmental bone defect reconstruction by Ilizarov type bone transport in an induced membrane. Orthop Traumatol Surg Res 2010;96(2):194-198

16 Ilizarov GA. Principes fondamentaux de l'ostéosynthèse en compression-distraction. Ortop Travmatol Protez 1971;32(11):7-15

17 Cattaneo R, Villa A, Catagni M, Tentori L. Traitement des pseudarthroses septiques ou non septiques selon la méth- ode d'Ilizarov en compression monofocale. Rev Chir Orthop Repar Appar Mot 1985;71:223-239

18 Weiland AJ, Moore JR, Daniel RK. Vascularized bone autografts. Experience with 41 cases. Clin Orthop Relat Res 1983;(174):87-95

19 Plakseychuk AY, Kim SY, Park BC, Varitimidis SE, Rubash HE, Sotereanos DG. Vascularized compared with nonvascularized fibular grafting for the treatment of osteonecrosis of the femoral head. J Bone Joint Surg Am 2003;85-A(4):589-596

20 Tetik C, Başar H, Bezer M, Erol B, Ağir I, Esemenli T. Comparison of early results of vascularized and non-vascularized fibular grafting in the treatment of osteonecrosis of the femoral head. Acta Orthop Traumatol Turc 2011;45(5):326-334

21 Kim SY, Kim YG, Kim PT, Ihn JC, Cho BC, Koo KH. Vascularized compared with nonvascularized fibular grafts for large osteonecrotic lesions of the femoral head. J Bone Joint Surg Am 2005;87(9):2012-2018
22 Taylor GI, Miller GD, Ham FJ. The free vascularized bone graft. A clinical extension of microvascular techniques. Plast Reconstr Surg 1975;55(5):533-544

23 Chen CM, Disa JJ, Lee HY, et al. Reconstruction of extremity long bone defects after sarcoma resection with vascularized fibula flaps: a 10-year review. Plast Reconstr Surg 2007;119(3):915924, discussion 925-926

24 Beris AE, Lykissas MG, Korompilias AV, et al. Vascularized fibula transfer for lower limb reconstruction. Microsurgery 2011;31(3):205-211

25 del Piñal F, Innocenti M. Evolving concepts in the management of the bone gap in the upper limb. Long and small defects. J Plast Reconstr Aesthet Surg 2007;60(7):776-792

26 Capanna R, Bufalini C, Campanacci M. A new technique for reconstructions of large metadiaphyseal bone defects, a combined graft (Allograft shell plus vascularised fibula). Orthop Traumatol Surg Res 1993;2(3):159-177

27 Taguchi Y, Pereira BP, Kour AK, Pho RW, Lee YS. Autoclaved autograft bone combined with vascularized bone and bone marrow. Clin Orthop Relat Res 1995;(320):220-230

28 Puhaindran ME, Pho RW. Biological reconstruction for children with osteosarcoma around the knee. Ann Acad Med Singapore 2014;43(10):499-505

29 Schwartz AJ, Kabo JM, Eilber FC, Eilber FR, Eckardt JJ. Endoprosthetic reconstruction after resection of musculoskeletal tumors. Am J Orthop 2014;43(3):122-127

30 Hanna SA, Sewell MD, Aston WJ, et al. Femoral diaphyseal endoprosthetic reconstruction after segmental resection of primary bone tumours.JBone JointSurg Br 2010;92(6):867-874

31 Aldlyami E, Abudu A, Grimer RJ, Carter SR, Tillman RM. Endoprosthetic replacement of diaphyseal bone defects. Long-term results. Int Orthop 2005;29(1):25-29

32 Fuchs B, Ossendorf C, Leerapun $\mathrm{T}$, Sim FH. Intercalary segmental reconstruction after bone tumor resection. Eur J Surg Oncol 2008;34(12):1271-1276

33 Rabitsch K, Maurer-Ertl W, Pirker-Frühauf U, Wibmer C, Leithner A. Intercalary reconstructions with vascularised fibula and allograft after tumour resection in the lower limb. Sarcoma 2013;2013:160295

34 Ruggieri P, Mavrogenis AF, Bianchi G, Sakellariou VI, Mercuri $\mathrm{M}$, Papagelopoulos PJ. Outcome of the intramedullary diaphyseal segmental defect fixation system for bone tumors. J Surg Oncol 2011;104(1):83-90

35 Sauerbruch VF. Dies exstirpation des femur mit Umkipp-Plastik des Unterschenkels. Deutsche Zeitschrift fur Chirugie 1922;169:1

36 Salzer M, Knahr K, Kotz R, Kristen H. Treatment of osteosarcomata of the distal femur by rotation-plasty. Arch Orthop Trauma Surg 1981;99(2):131-136

37 Hopyan S, Tan JW, Graham HK, Torode IP. Function and upright time following limb salvage, amputation, and rotationplasty for pediatric sarcoma of bone. J Pediatr Orthop 2006;26(3):405-408

38 Catani F, Capanna R, Benedetti MG, et al. Gait analysis in patients after Van Nes rotationplasty. Clin Orthop Relat Res 1993;(296):270-277

39 Gottsauner-Wolf F, Kotz R, Knahr K, Kristen H, Ritschl P, Salzer M. Rotationplasty for limb salvage in the treatment of malignant tumors at the knee. A follow-up study of seventy patients. J Bone Joint Surg Am 1991;73(9):1365-1375

40 Nishinari K, Wolosker N, Yazbek G, Zerati AE, Nishimoto IN. Venous reconstructions in lower limbs associated with resection of malignancies. J Vasc Surg 2006;44(5):1046-1050

41 Nishinari K, Wolosker N, Yazbek G, et al. Vascular reconstruction in limbs with malignant tumors. Vasc Endovascular Surg 2004;38(5):423-429

42 Nishinari K, Krutman M, Aguiar Junior S, et al. Surgical outcomes of vascular reconstruction in soft tissue sarcomas of the lower extremities. J Vasc Surg 2015;62(1):143-149 
43 Tsukushi S, Nishida Y, Sugiura H, Nakashima H, Ishiguro N. Results of limb-salvage surgery with vascular reconstruction for soft tissue sarcoma in the lower extremity: comparison between only arterial and arterovenous reconstruction. J Surg Oncol 2008;97(3):216-220

44 Kawai A, Hashizume H, Inoue H, Uchida H, Sano S. Vascular reconstruction in limb salvage operations for soft tissue tumors of the extremities. Clin Orthop Relat Res 1996; (332):215-222

45 Pinsolle V, Reau AF, Pelissier P, Martin D, Baudet J. Soft-tissue reconstruction of the distal lower leg and foot: are free flaps the only choice? Review of 215 cases. J Plast Reconstr Aesthet Surg 2006;59(9):912-917, discussion 918

46 Parrett BM, Matros E, Pribaz JJ, Orgill DP. Lower extremity trauma: trends in the management of soft-tissue reconstruction of open tibia-fibula fractures. Plast Reconstr Surg 2006;117(4):1315-1322, discussion 1323-1324

47 Cordeiro PG, Neves RI, Hidalgo DA. The role of free tissue transfer following oncologic resection in the lower extremity. Ann Plast Surg 1994;33(1):9-16

48 Lin CH, Lin YT, Yeh JT, Chen CT. Free functioning muscle transfer for lower extremity posttraumatic composite structure and functional defect. Plast Reconstr Surg 2007;119(7):2118-2126

49 Wechselberger G, Pichler M, Pülzl P, Schoeller T. Free functional rectus femoris muscle transfer for restoration of extension of the foot after lower leg compartment syndrome. Microsurgery 2004;24(6):437-441

50 Hallock GG. Restoration of quadriceps femoris function with a dynamic microsurgical free latissimus dorsi muscle transfer. Ann Plast Surg 2004;52(1):89-92
51 Willcox TM, Smith AA, Beauchamp C, Meland NB. Functional free latissimus dorsi muscle flap to the proximal lower extremity. Clin Orthop Relat Res 2003;(410):285-288

52 Ihara K, Shigetomi M, Kawai S, Doi K, Yamamoto M. Functioning muscle transplantation after wide excision of sarcomas in the extremity. Clin Orthop Relat Res 1999;(358):140-148

53 Kobayashi S, Sekiguchi J, Sakai Y, Ohmori K. Functioning free muscle transplantation to the lower leg. J Reconstr Microsurg 1995;11(5):319-325

54 Markhede G, Stener B. Function after removal of various hip and thigh muscles for extirpation of tumors. Acta Orthop Scand 1981;52(4):373-395

55 Goldthwait JE. The direct transplantation of muscles in the treatment of paralytic deformities. Trans Am Orthop Assoc 1897; 10:246-252

56 Pritsch T, Malawer MM, Wu CC, Squires MH, Bickels J. Functional reconstruction of the extensor mechanism following massive tumor resections from the anterior compartment of the thigh. Plast Reconstr Surg 2007;120(4):960-969

57 Dubousset J, Missenard G, Genin J. [Conservative surgical treatment of osteogenic sarcoma of the limbs. Technics and functional results]. Rev Chir Orthop Repar Appar Mot 1985;71(7):435-450

58 Muramatsu K, Ihara K, Miyoshi T, Yoshida K, Hashimoto T, Taguchi T. Transfer of latissimus dorsi muscle for the functional reconstruction of quadriceps femoris muscle following oncological resection of sarcoma in the thigh. J Plast Reconstr Aesthet Surg 2011;64(8):1068-1074 